\title{
Using Pre-TMIn Treatment to Improve the Optical Properties of Green Light Emitting Diodes
}

\author{
Bing Xu, ${ }^{1,2}$ Hai Tao Dai, ${ }^{1}$ Shu Guo Wang, ${ }^{1}$ Fu-Chuan Chu, ${ }^{2}$ Chou-Hsiung Huang, \\ Sheng-Fu Yu, ${ }^{3}$ Jun Liang Zhao, ${ }^{4}$ Xiao Wei Sun, ${ }^{5}$ and Ray-Ming Lin ${ }^{2}$ \\ ${ }^{1}$ Tianjin Key Laboratory of Low Dimensional Materials Physics and Preparing Technology, School of Science, \\ Tianjin University, Tianjin 300072, China \\ ${ }^{2}$ Graduate Institute of Electronic Engineering and Green Technology Research Center, Chang Gung University, Taoyuan 333, Taiwan \\ ${ }^{3}$ Institute of Microelectronics and Department of Electrical Engineering, Center for Micro/Nano Science and Technology, \\ Advanced Optoelectronic Technology Center, National Cheng Kung University, Tainan 70101, Taiwan \\ ${ }^{4}$ Shanghai Juntech Co. Ltd., 1378 Xingxian Road, Shanghai 201815, China \\ ${ }^{5}$ Centre of Excellence for Semiconductor Lighting and Displays, School of Electrical and Electronic Engineering, \\ Nanyang Technological University, 50 Nanyang Avenue, Singapore 639798
}

Correspondence should be addressed to Ray-Ming Lin; rmlin@mail.cgu.edu.tw

Received 16 April 2014; Revised 6 June 2014; Accepted 24 June 2014; Published 10 July 2014

Academic Editor: Sheng-Po Chang

Copyright (C) 2014 Bing Xu et al. This is an open access article distributed under the Creative Commons Attribution License, which permits unrestricted use, distribution, and reproduction in any medium, provided the original work is properly cited.

\begin{abstract}
We investigated the effects of pre-TMIn treatment on the optical properties of green light emitting diodes (LEDs). Although pre-TMIn treatment did not affect the epitaxial structure of quantum wells, it significantly improved the quality of the surface morphology relative to that of the untreated sample. Indium cluster can be seen by high-resolution transmission electron microscopy (HR-TEM), which is the explanation for the red-shift of photoluminescence (PL). Time-resolved photoluminescence measurements indicated that the sample prepared with pre-TMIn treatment had a shorter radiative decay time. As a result, the light output power of the treated green LED was higher than that of the conventional untreated one. Thus, pre-TMIn treatment appears to be a simple and efficient means of improving the performance of green LEDs.
\end{abstract}

\section{Introduction}

Group III-nitride materials have attracted much interest for their use in optoelectronic applications because their emission spectra cover a wide range from the infrared to the deep ultraviolet [1-3]. Among them, indium gallium nitride (InGaN) is the most promising material for the preparation of high-efficiency, high-power light emitting diodes (LEDs), which are employed widely in backlighting, traffic lights, headlights, and general illumination [4]. Although InGaNbased blue LEDs are well established, the InGaN-based green LEDs continue to suffer from low efficiency, which limits their further applications in white lighting devices.

V-shaped defects [5-7] readily form at InGaN/GaN quantum wells (QWs) having high indium mole fractions, triggered by threading dislocations in the buffer layer. Several factors can lead to a low-quality InGaN layer, including lattice mismatch between InN and GaN [8], low miscibility of InN $[9,10]$, phase separation [11], indium surface segregation [12], and V-shaped defects [5-7]. A growth interruption technique $[13,14]$ can be used to improve the quality of InGaN layers and to enhance the luminescence intensity of LED devices. By applying growth interruption at a high growth temperature, the atoms in the InGaN layer can migrate to their minimum energy sites to approach thermal equilibrium and decomposition of In-rich regions, leading to a flat InGaN surface. Another growth method in which trimethylindium (TMIn) is employed during the growth interruption process is known as "TMIn treatment" [15]; it can suppress InGaN decomposition and indium aggregation to achieve a more homogeneous indium composition, a lower density of V-shaped defects, higher energy states, stronger photoluminescence intensity, 


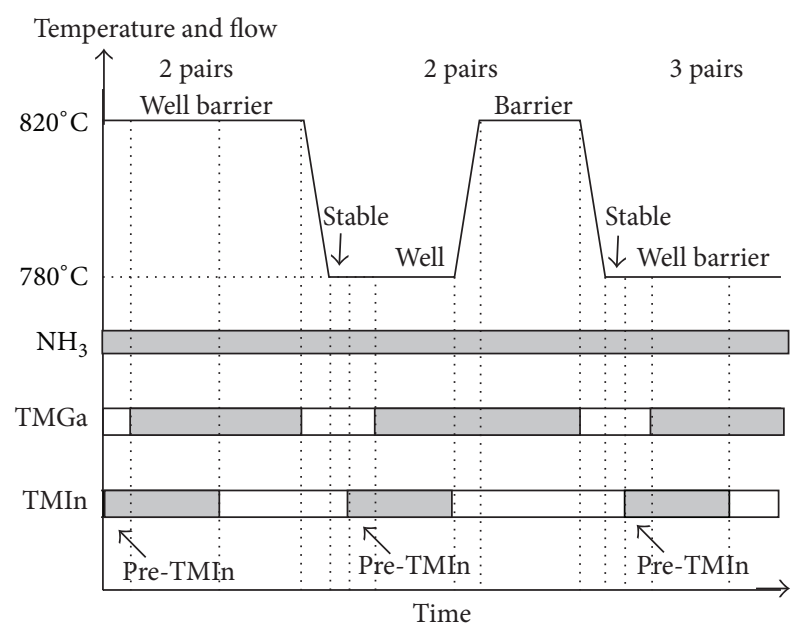

FIGURE 1: Growth conditions for pre-TMIn treatment in InGaN/ GaN MQW.

and shorter decay times. In this study, we examined the effects of pre-TMIn treatment prior to QW growth on the output power of InGaN-based green LEDs. Our experimental results revealed that the light output intensity from a sample subjected to pre-TMIn treatment exceeded that of conventional LEDs at a quite high injection level, a result of stronger quantum confinement ability arising from indium-rich sites. Time-resolved photoluminescence (TRPL) revealed that the radiative recombination effect of the TMIn-treated sample was better than that of its untreated counterpart.

\section{Experimental Methods}

InGaN/GaN multiple quantum well (MQW) samples were grown on a (0001) sapphire substrate using an atmospheric pressure metal-organic chemical vapor deposition system. Trimethylgallium, trimethylindium, trimethylaluminum, and ammonia $\left(\mathrm{NH}_{3}\right)$ are used as precursors of the $\mathrm{Ga}$, In, $\mathrm{Al}$, and $\mathrm{N}$ atoms, respectively. Bicyclopentadienyl and silane are used as the $\mathrm{p}$ - and n-type dopant precursors. The substrates were first heated in $\mathrm{H}_{2}$ ambient to remove any contaminants prior to performing the growth process. The LED structures comprised a $25 \mathrm{~nm}$ low-temperature GaN nucleation layer; a $1 \mu \mathrm{m}$ unintentionally doped $\mathrm{GaN}$ buffer layer; a $3 \mu \mathrm{m} \mathrm{n}$-GaN layer; seven periods of MQW layers featuring $2.7 \mathrm{~nm}$ InGaN well layers and $8 \mathrm{~nm}$ GaN barrier layers; a $10 \mathrm{~nm}$ AlGaN electron blocking layer; and a $100 \mathrm{~nm}$ $\mathrm{p}-\mathrm{GaN}$ layer. There are two samples which are without and with pre-TMIn flow and each sample has three sets of MQW layers. The detail of pre-TMIn flow condition is (i) two pairs of InGaN QWs and $\mathrm{GaN}$ barriers grown at $820^{\circ} \mathrm{C}$ with a TMIn preflow temperature of $820^{\circ} \mathrm{C}$; (ii) three pairs of InGaN QWs and $\mathrm{GaN}$ barriers grown at 780 and $820^{\circ} \mathrm{C}$, respectively, with a TMIn preflow temperature of $780^{\circ} \mathrm{C}$; and (iii) three pairs of InGaN QWs and $\mathrm{GaN}$ barriers grown at $780^{\circ} \mathrm{C}$ with a TMIn preflow temperature of $780^{\circ} \mathrm{C}$. Before the deposition of the InGaN QW layers, only TMIn and $\mathrm{NH}_{3}$ were flowed into the reactor. For easy understanding, the details are schematically

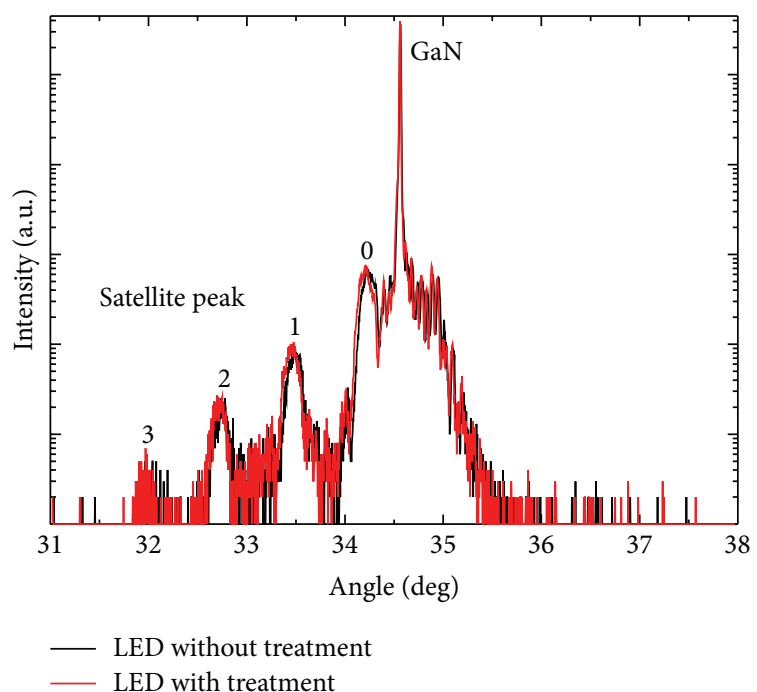

FIGURE 2: XRD spectra for the LEDs prepared with and without TMIn treatment.

represented in Figure 1. The final LEDs were fabricated, by means of standard LED process technology, to have an active area of $1 \times 1 \mathrm{~mm}^{2}$.

The epitaxial wafers were subjected to analysis through high-resolution X-ray diffraction (HR-XRD), high-resolution transmission electron microscopy (HR-TEM), atomic force microscopy (AFM), TRPL, and room-temperature photoluminescence; the light output power was measured to characterize the optical properties of the LED devices.

\section{Results and Discussion}

Figure 2 displays the HR-XRD spectra of the LEDs prepared with and without pre-TMIn treatment. The diffraction peak of $\mathrm{GaN}$ is identified and marked in the curve; it arose from the buffer and the n- and p-type contact layers. We used Vegard's law to calculate the indium composition from the lattice parameters determined through XRD. The XRD curves of the two samples are almost identical, implying that the structure of the MQWs did not change as a result of TMIn treatment, except for a slight increase in the indium content in the InGaN layer. The indium compositions are $24.5 \%$ and $25 \%$ for the samples without and with pre-TMIn treatment, respectively. The satellite peaks suggested good quality interfaces for the MQW structures. Our results confirm that indium diffusion into the barrier could be suppressed and that the indium composition became homogeneous after TMIn treatment [15].

We used AFM and SEM to observe the surface morphologies of the two samples (Figure 3). The surface morphology of the sample that had experienced pre-TMIn treatment was better than that of the untreated sample, with the root-meansquare (RMS) surface roughness observed decreasing from 0.819 to $0.708 \mathrm{~nm}$. Figures 3(c) and 3(d) show the surface morphology of InGaN QWs layer etched by phosphoric acid $\left(\mathrm{H}_{3} \mathrm{PO}_{4}\right)$ for $30 \mathrm{~s}$ at $220^{\circ} \mathrm{C}$. The etch pits density is about 1.1 $\times 10^{9} \mathrm{~cm}^{-2}$ and $7.3 \times 10^{8} \mathrm{~cm}^{-2}$ for the sample without and 


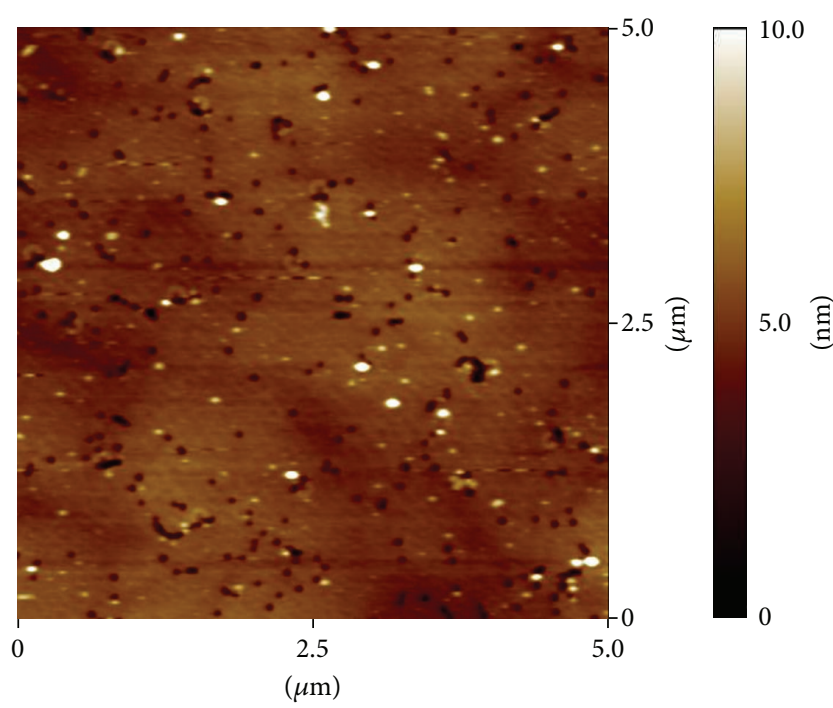

(a)

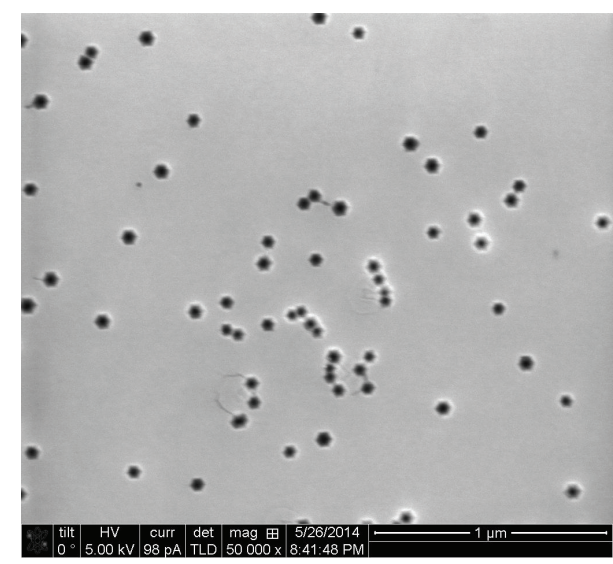

(c)

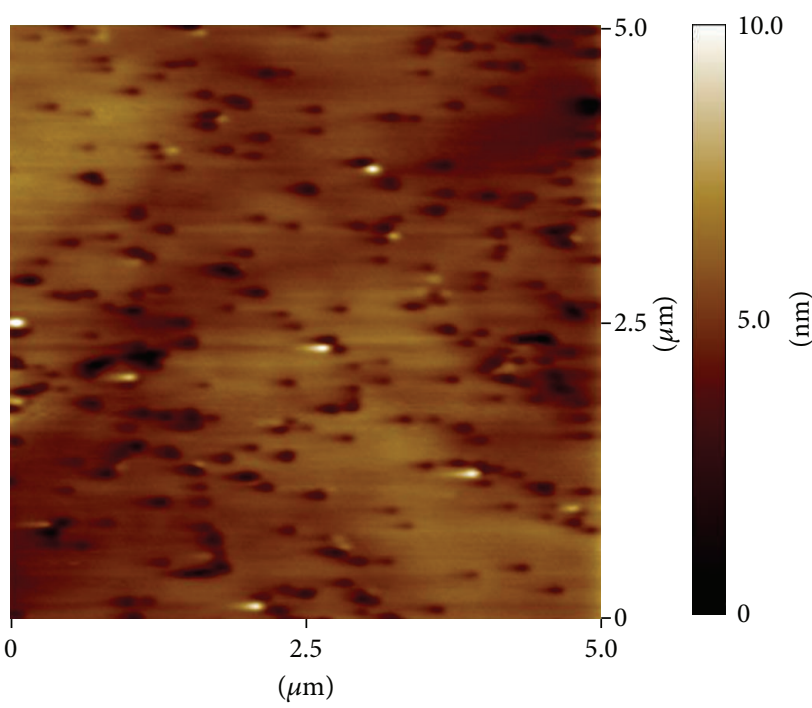

(b)

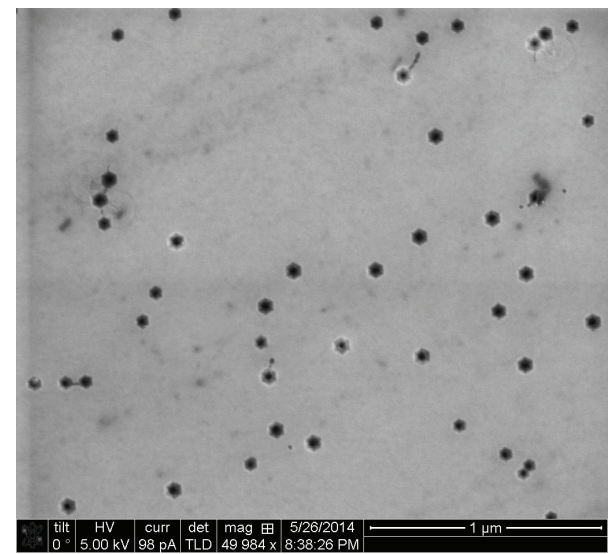

(d)

FIGURE 3: AFM and SEM images of the surface morphologies of the (a), (c) sample without TMIn treatment and (b), (d) sample with TMIn treatment.

with TMIn treatment, respectively. This finding suggested that TMIn treatment could improve the quality of the InGaNGaN interface, thereby potentially improving the optical properties of green LEDs.

We used room-temperature photoluminescence to determine the center wavelengths of the two samples; Figure 4 displays their spectra. Photoluminescence peaks for the untreated and treated samples appeared at 520 and $535 \mathrm{~nm}$, respectively; that is, a red-shift in the signal occurred after TMIn treatment. In general, such a red-shift occurs upon increasing the mole fraction of indium. Our XRD profiles in Figure 2 reveal, however, that the indium content increased only slightly. Considering the material properties of indium, we believe that the red-shift was related to aggregation of indium atoms in the QW during TMIn treatment $[15,16]$. The action of TMIn and $\mathrm{NH}_{3}$ could not form a continuous InN film, but rather InN islands, during the short treatment time and under the high growth temperature. In order to prove our thought, HR-TEM measurement was employed. Figure 5 presents the cross section HR-TEM images for two samples (a) without TMIn treatment, (b) with TMIn treatment. From Figures 5(a) and 5(b), the barriers and wells can be easily distinguished. As we can see, the dark dots in Figure 5(b) are much more than in Figure 5(a). These dark points are In-rich cluster and their formation can be explained by pre-TMIn flow treatment, which is the main reason for the red-shift of PL.

We employed TRPL to examine the recombination rate of green LEDs that had been prepared with pre-TMIn treatment. We fitted the PL decay profiles in Figure 6 with the following double-exponential equation to obtain the decay time:

$$
I(t)=A_{1} \exp \left(-\frac{t}{\tau_{r}}\right)+A_{2} \exp \left(-\frac{t}{\tau_{\mathrm{nr}}}\right),
$$

where $I(t)$ is the PL intensity, $A_{1}$ and $A_{2}$ are the decay parameters, and $\tau_{r}$ and $\tau_{\mathrm{nr}}$ are the decay times for radiative and nonradiative recombination [17]. For the treated sample, the values of $\tau_{r}$ and $\tau_{\mathrm{nr}}$ were $6.2 \times 10^{-12}$ and $3.6 \times 10^{-9} \mathrm{~s}$, respectively; for the untreated sample, they were $9.1 \times 10^{-12}$ 


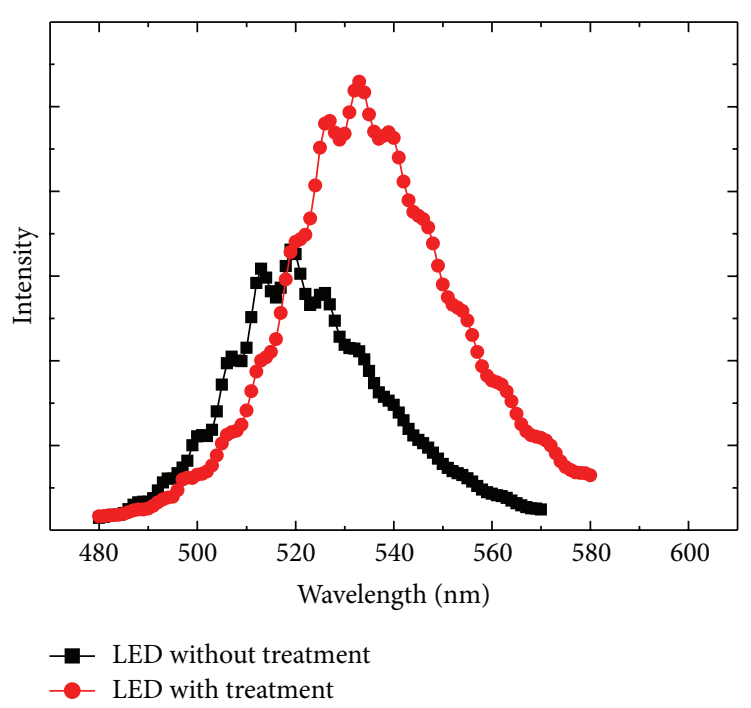

FIGURE 4: Room-temperature photoluminescence spectra for the LEDs prepared without and with TMIn treatment, with central peaks located at 520 and $535 \mathrm{~nm}$, respectively.

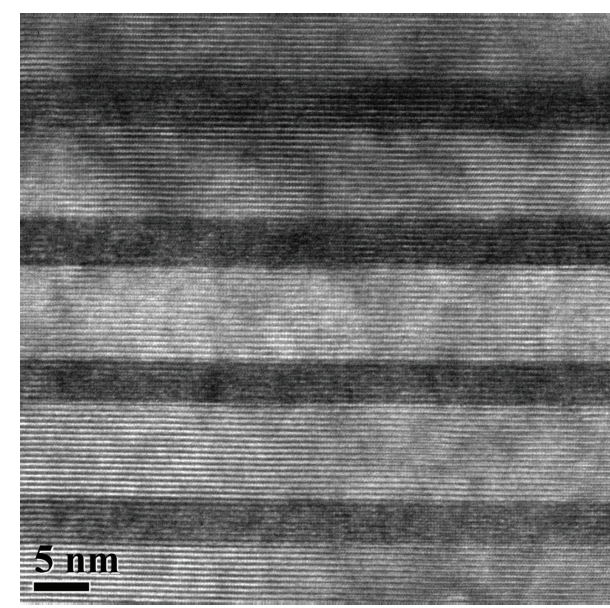

(a)

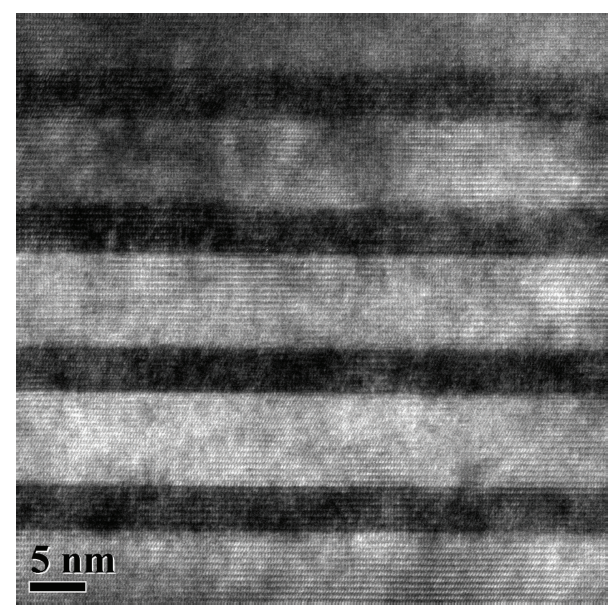

(b)

FIGURE 5: HR-TEM images of MQWs for two samples, (a) without TMIn treatment and (b) with TMIn treatment.

and $3.4 \times 10^{-9} \mathrm{~s}$, respectively. Thus, the radiative decay time decreased as a result of pre-TMIn treatment, leading to a greater radiative recombination effect. Because carrier transport into weakly localized states requires a certain energy to overcome a potential barrier, it is more difficult for carriers to transfer into weakly localized states $[17,18]$. In the localized exciton model, trap centers are originated from a spatial disorder such as the fluctuation of indium within InGaN/GaN MQWs. Using TMIn treatment, the decay time becomes shorter, because treatment leads to more strongly localized stats due to the indium cluster [18].

Figure 7 plots the light output power $(L)$ and the voltage $(V)$ with respect to the injection current $(I)$ for the pre-TMIntreated and untreated LEDs. The treated sample exhibited higher output power throughout the whole injection current density. At an injection current of $350 \mathrm{~mA}$, the light output power of the treated LED was 59\% higher than that of the untreated LED and the forward voltages are 3.66 and $3.68 \mathrm{~V}$ for the samples without and with pre-TMIn treatment. We attribute this enhancement in light output power to the presence of In-rich clusters in the pre-TMIn-treated sample; these clusters could confine electrons and holes efficiently. As a result, indium treatment plays an important role in improving the luminescence of green LEDs.

\section{Conclusion}

Pre-TMIn treatment of the InGaN layer appears to be an efficient means of improving the luminescence performance of an InGaN green LED because it leads to a smoother surface and a shorter radiative decay time. XRD revealed that pre-TMIn treatment did not change the structure of the 


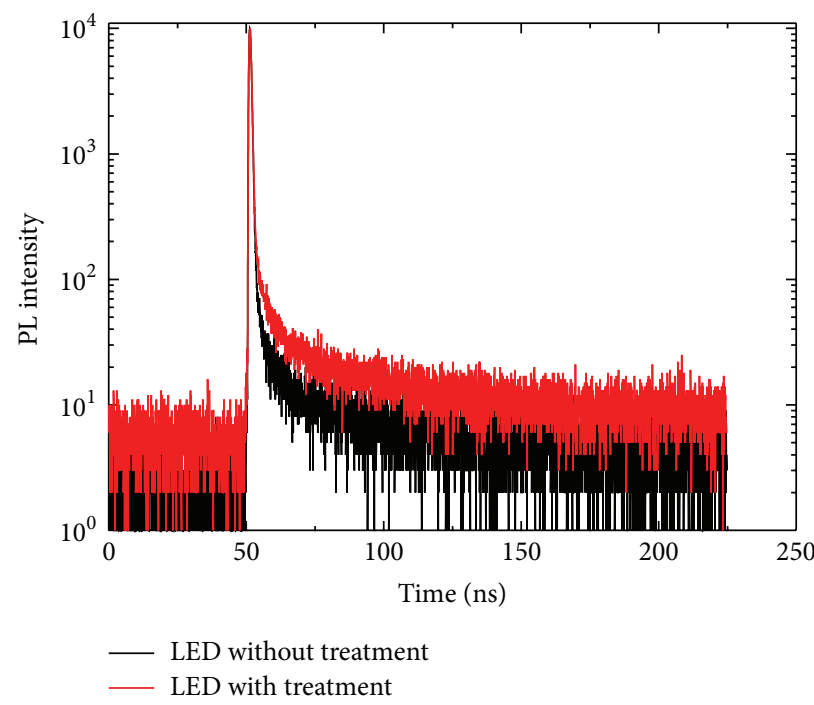

FIGURE 6: Time-resolved photoluminescence decay profiles of the LEDs prepared with and without TMIn treatment.

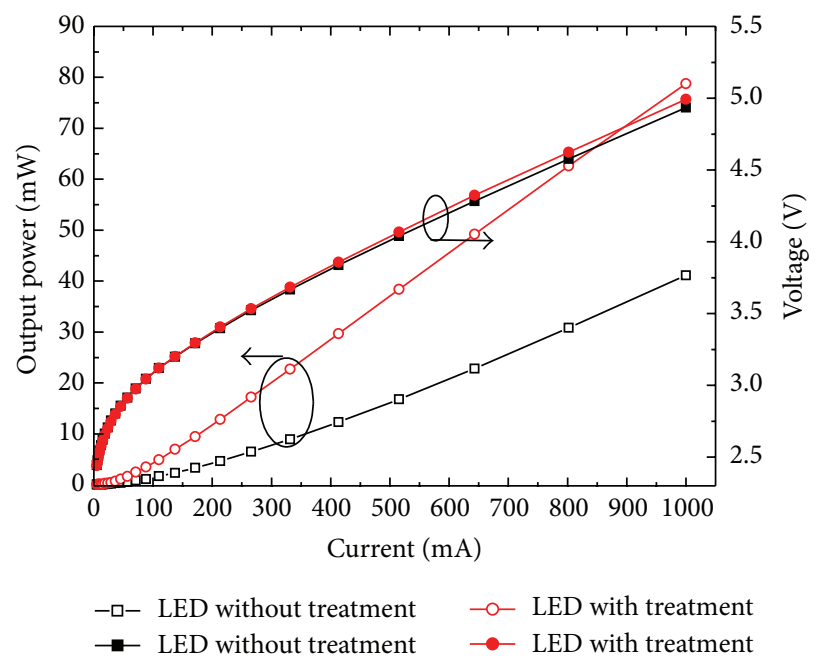

FIGURE 7: Plots of light output power and forward voltage with respect to injection current for the LEDs prepared with and without TMIn treatment.

MQWs and only slightly increased the indium concentration. Photoluminescence spectra of the treated sample featured a red-shifted peak wavelength, due to enhancement of the indium-cluster, which can be seen by HR-TEM. In addition, the light output power improved significantly when the LED was prepared with pre-TMIn treatment of the InGaN layer.

\section{Conflict of Interests}

The authors declare that there is no conflict of interests regarding the publication of this paper.

\section{Acknowledgments}

This study was supported by the National Natural Science Foundation of China (NSFC; Project nos. 61177061, 61177014, and 61076015), the Tianjin Natural Science Foundation (Project nos. 11JCZDJC21900 and 11JCYBJC25800), the Research Fund for the Doctoral Program of Higher Education of China (New Teacher) (under Grant no. 20110032120070), the Special Foundation for Nanotechnology of the Shanghai Committee for Science and Technology (no. 11nm0501500), the Shanghai Pujiang Program (no. 12PJ1431000), and the Open Project Program of State Key Laboratory of Precision Measuring Technology and Instruments (Tianjin University).

\section{References}

[1] S. C. Jain, M. Willander, J. Narayan, and R. van Overstraeten, "III-nitrides: growth, characterization, and properties," Journal of Applied Physics, vol. 87, no. 3, pp. 965-1006, 2000.

[2] S. Nakamura, M. Senoh, S. Nagahame et al., "InGaN/GaN/ AlGaN-based laser diodes with modulation-doped strainedlayer superlattices," Japanese Journal of Applied Physics, vol. 36, no. 12, pp. L1568-L1571, 1997. 
[3] R. M. Lin, M. J. Lai, L. B. Chang, C. H. Huang, and C. H. Chen, "Effect of trapezoidal-shaped well on efficiency droop in InGaN-based double-heterostructure light-emitting diodes," International Journal of Photoenergy, vol. 2012, Article ID 917159, 5 pages, 2012.

[4] T. Erdem, S. Nizamoglu, X. W. Sun, and H. V. Demir, "A photometric investigation of ultra-efficient LEDs with high color rendering index and high luminous efficacy employing nanocrystal quantum dot luminophores," Optics Express, vol. 18, no. 1, pp. 340-347, 2010.

[5] T. Hino, S. Tomiya, T. Miyajima, K. Yanashima, S. Hashimoto, and M. Ikeda, "Characterization of threading dislocations in GaN epitaxial layers," Applied Physics Letters, vol. 76, no. 23, pp. 3421-3423, 2000.

[6] S. F. Yu, S. P. Chang, S. J. Chang, R. M. Lin, H. H. Wu, and W. C. Hsu, "Characteristics of InGaN-based light-emitting diodes on patterned sapphire substrates with various pattern heights," Journal of Nanomaterials, vol. 2012, Article ID 346915, 6 pages, 2012.

[7] H. Saijo, J. T. Hsu, R. C. Tu et al., "Mapping of multiple-quantum-well layers and structure of $\mathrm{V}$ defects in InGaN/GaN diodes," Applied Physics Letters, vol. 84, no. 13, pp. 2271-2273, 2004.

[8] T. Takeuchi, C. Wetzel, S. Yamaguchi et al., "Determination of piezoelectric fields in strained GaInN quantum wells using the quantum-confined Stark effect," Applied Physics Letters, vol. 73, no. 12, pp. 1691-1693, 1998.

[9] Y. Lin, K. Ma, C. Hsu et al., "Dependence of composition fluctuation on indium content in InGaN/GaN multiple quantum wells," Applied Physics Letters, vol. 77, no. 19, pp. 2988-2990, 2000.

[10] S. F. Chichibu, A. Uedono, T. Onuma et al., "Origin of defectinsensitive emission probability in In-containing (Al,In, Ga)N alloy semiconductors," Nature Materials, vol. 5, no. 10, pp. 810816, 2006.

[11] Y. Narukawa, Y. Kawakami, M. Funato, S. Fujita, and S. Nakamura, "Role of self-formed InGaN quantum dots for exciton localization in the purple laser diode emitting at $420 \mathrm{~nm}$," Applied Physics Letters, vol. 70, no. 8, pp. 981-983, 1997.

[12] H. K. Cho, J. Y. Lee, G. M. Yang, and C. S. Kim, "Formation mechanism of $\mathrm{V}$ defects in the InGaN/GaN multiple quantum wells grown on $\mathrm{GaN}$ layers with low threading dislocation density," Applied Physics Letters, vol. 79, no. 2, pp. 215-217, 2001.

[13] S. Y. Kwon, H. J. Kim, H. Na et al., "Effect of growth interruption on In-rich InGaN/GaN single quantum well structures," Physica Status Solidi (C), no. 7, pp. 2830-2833, 2003.

[14] H. K. Cho, J. Y. Lee, N. Sharma, J. Humphreys, G. M. Yang, and C. S. Kim, "Structural and optical characteristics of InGaN/GaN multiple quantum wells with different growth interruption," physica Status Solidi (b), vol. 228, no. 1, pp. 165-168, 2001.

[15] M. Senthil Kumar, J. Y. Park, Y. S. Lee, S. J. Chung, C. Hong, and E. Suh, "Improved internal quantum efficiency of green emitting In GaN/GaN multiple quantum wells by in preflow for InGaN well growth," Japanese Journal of Applied Physics, vol. 47, no. 2, pp. 839-842, 2008.

[16] S. Leem, M. H. Kim, J. Shin, Y. Choi, and J. Jeong, “The effects of in flow during growth interruption on the optical properties of InGaN multiple quantum wells grown by low pressure metalorganic chemical vapor deposition," Japanese Journal of Applied Physics, vol. 40, no. 2, pp. L371-L373, 2001.

[17] Y. B. Tao, Z. Z. Chen, T. J. Yu et al., "Improvement of structural and luminescence properties in InGaN/GaN multiple quantum wells by symmetrical thin low temperature-GaN layers," Journal of Crystal Growth, vol. 318, no. 1, pp. 509-512, 2011.

[18] S. Feng and J. Chyi, "Carrier transport study of TMIn-treated InGaN LEDs by using quantum efficiency and time-resolved electro-luminescence measurements," Journal of the Electrochemical Society, vol. 159, no. 3, pp. H225-H229, 2012. 

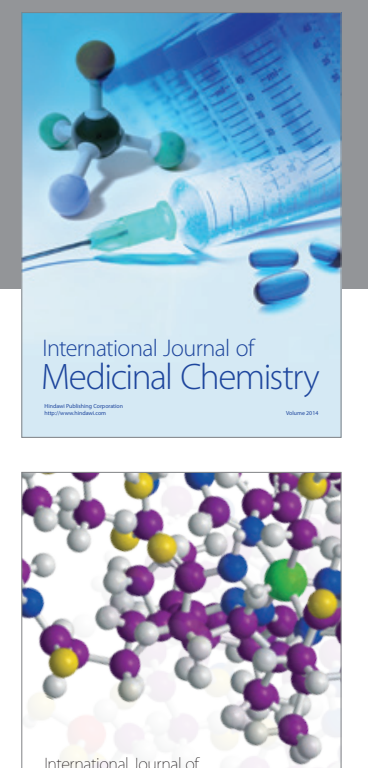

\section{Carbohydrate} Chemistry

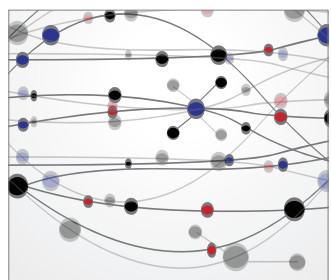

The Scientific World Journal
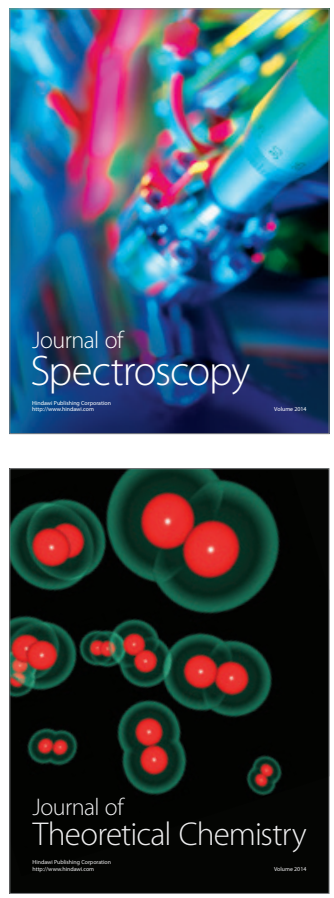
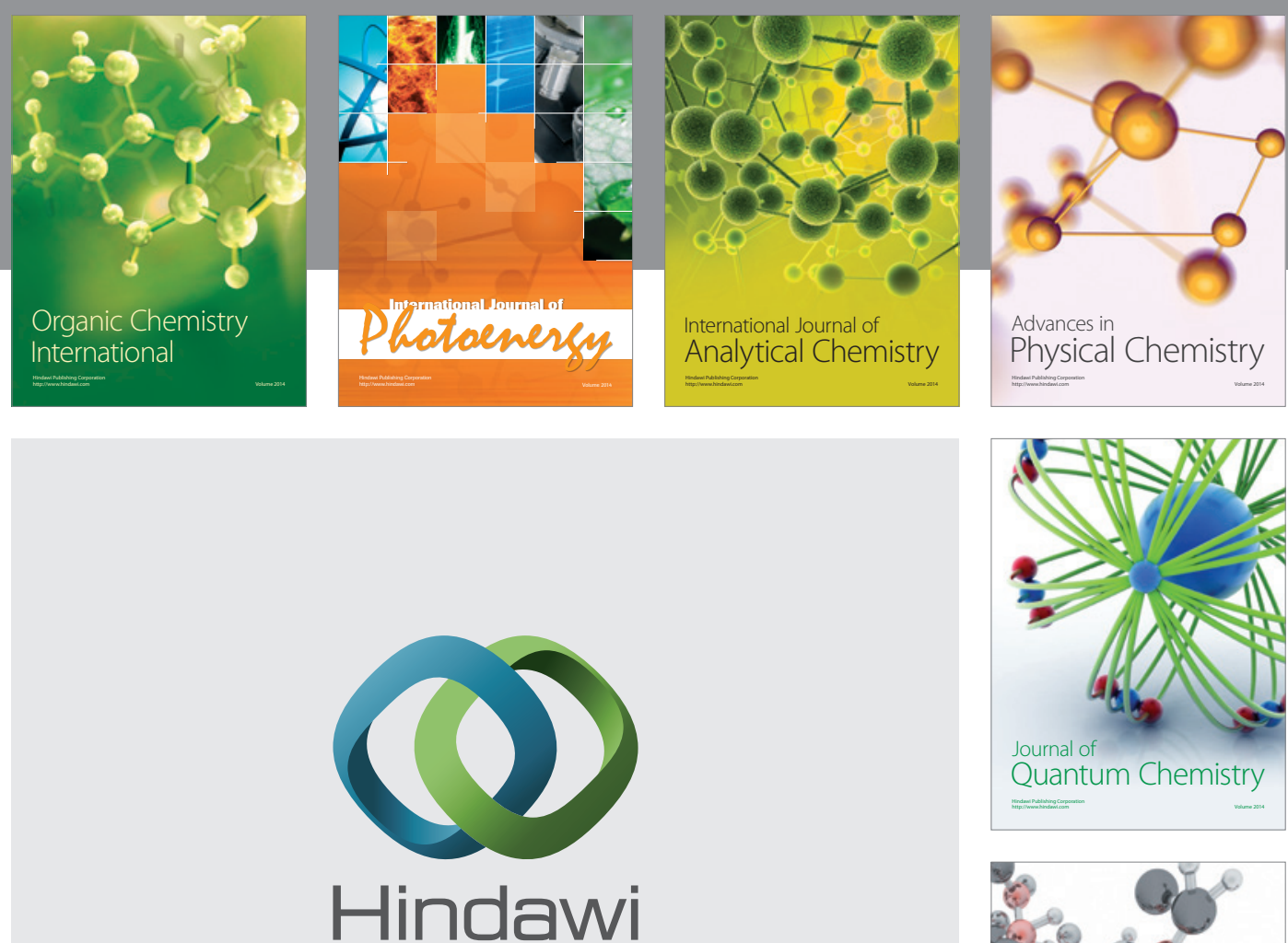

Submit your manuscripts at

http://www.hindawi.com

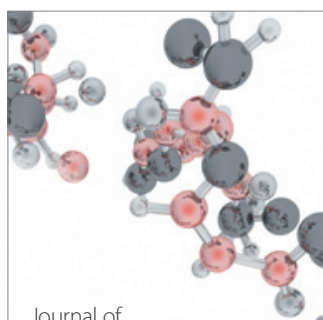

Analytical Methods

in Chemistry

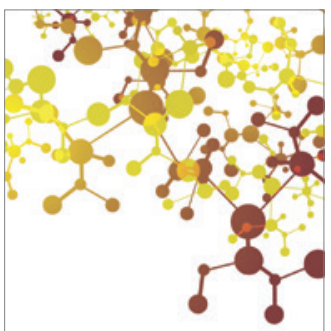

Journal of

Applied Chemistry

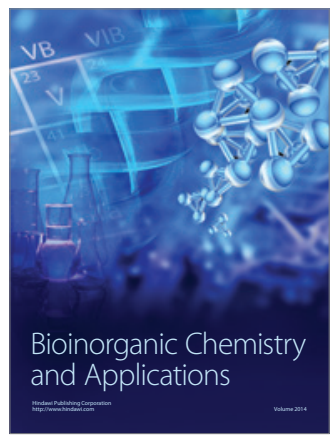

Inorganic Chemistry
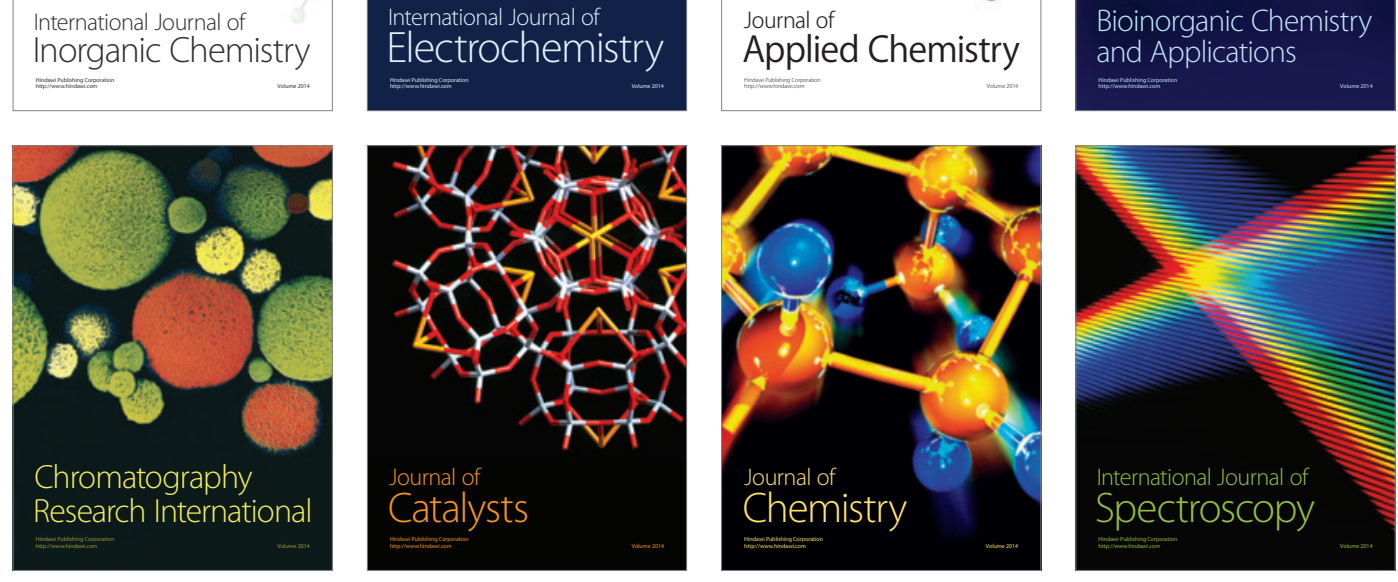